\title{
FEASIBILITY STUDY OF OPTICAL DETECTION OF CHATTER VIBRATION DURING MILLING
}

\author{
Andras Eppel, Eniko T. Enikov, Tamas Insperger, \\ and Stepan Gabor \\ Department of Aerospace and Mechanical Engineering, \\ University of Arizona, Tucson, Arizona, USA
}

This article introduces an optical device for measuring and recording the vibrations during milling. The constructed apparatus is able to discriminate between stable cutting and chatter vibration. A review of various chatter detection methods is presented. It lists basic numerical approximation methods to predict chatter and the devices used to detect unstable cutting. A discussion on the importance of experimental detection versus theoretical predictions is also included. The article further presents the measurement setup, its basic components, and their parameters along with the basic principles of the measurements and the theoretical framework of the stability analysis. The theoretical framework is then applied to show the chatter determining frequencies and to determine what has to be detected during the measurements. Experimental results for slotting and down milling with different immersion ratios are also presented. These include stability maps based on the power spectrum density (PSD) graphs of the collected data and confirmed by photographs of the cut surfaces. The conclusion summarizes the results, and describes advantages and disadvantages of the setup.

Keywords: chatter vibration, milling, optical detection

\section{INTRODUCTION}

Machining operations, like cutting, drilling, and milling are one of the most commonly used manufacturing processes. These have been carried out using experience-based approaches. The optimum cutting conditions are determined after extensive shop-floor tests over a long time. With the increase of industrial competition, the need of higher accuracy and lower production costs has become apparent. At the same time, the quality of the manufactured products has to be maintained. During a milling process, one of the most disturbing phenomena is the so-called chatter vibration. This is a dynamic instability resulting in low surface quality that can also lead to workpiece and tool damage. Self-excited vibrations of the cutter, holder, spindle, or machine cause chatter resulting in very large chip thickness variations as each tooth of the cutter passes through the cut. The vibration continues to grow until the chip thickness is exceeded and the cutter jumps out

Address correspondence to Eniko T. Enikov, Department of Aerospace and Mechanical Engineering, University of Arizona, 1130 N. Mountain Ave., Tucson, Arizona 85721, USA. E-mail: enikov@engr.arizona.edu 


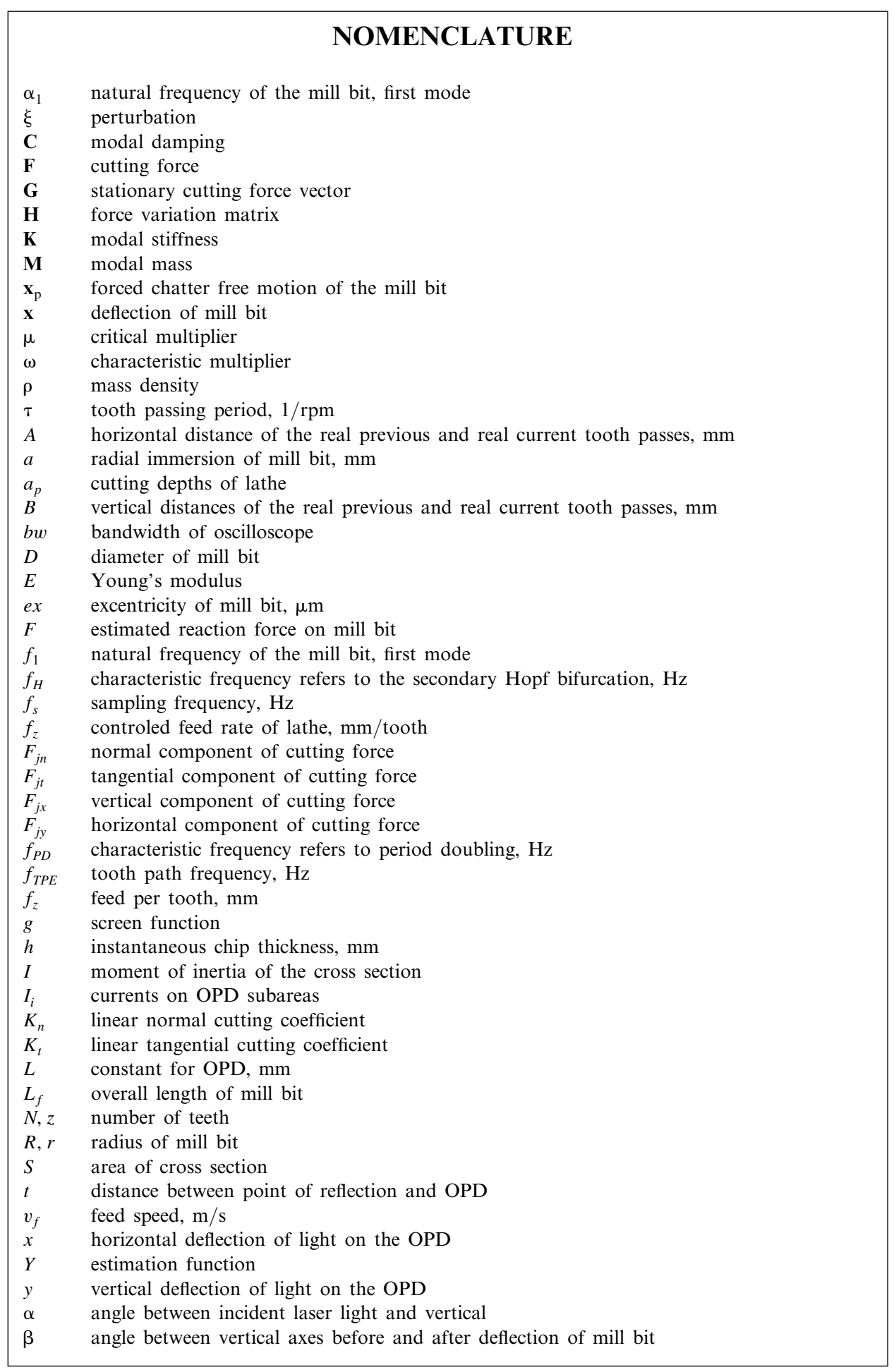




\begin{tabular}{|ll|}
\hline & \\
& \\
$\Delta R_{r}$ & NOMENCLATURE \\
$\Delta R_{t}$ & position resolution of OPD, $\mu \mathrm{m}$ \\
$\Delta s$ & deflection of laser beam on OPD \\
$\Delta s_{\max }$ & maximal deflection of laser beam on OPD \\
$\Delta x$ & deflection of mill bit \\
$\Omega$ & spindle speed of lathe, rpm \\
$\phi_{j}$ & angular position of the $j$ th cutting edge, rad \\
DDE & delay differential equation \\
FFT & fast Fourier transformation \\
LDS & laser distance triangulation sensor \\
LDV & laser doppler vibrometer \\
LI & laser interferometer \\
OPD & optical position detector \\
PSD & power spectrum density \\
SDM & semi-discretisation method \\
ZOA & zeroth order approximation \\
\hline
\end{tabular}

of the cut. In the past decade, extensive research was conducted to develop accurate dynamic machining models to map all of the stable and unstable cutting conditions. The work of Tlustý et al. (1962) and Tobias (1965) led to the development of the stability lobe diagram, that plots the boundary between stable and unstable cuts as a function of spindle speed. The stability analysis of such a system can only be performed by applying approximated numerical methods such as time-domain simulations, frequency-domain simulations, or delay-differential equation (DDE) based methods. Recently, several time-domain simulations of a milling process were proposed (Campomanes and Altintas 2003). For a given combination of cutting parameters, the vibrations of the milling process are numerically simulated by discretizing the process describing DDEs system with Euler, Runge Kutta, or Tustin's approximations. The stability of the system is assessed by evaluating the time behavior of the vibrations. This approach is very time consuming, and the applicability in the industry is limited. On the other hand, it is capable of modeling the effective kinematics of milling, and some important nonlinear effects. Therefore, the results of the time-domain simulations are often used as a reference for other methods. The first effective frequency-domain method was derived by Altintas and Budak (1995b). It is called zeroth order approximation (ZOA). The applicability of this method is limited to the slot milling configurations. It is not capable of representing highly intermittent milling processes with small radial immersions, whose cutting force trends require more harmonics to be approximated. Other methods for chatter prediction are based on the delay differential equation (DDE) theory. In 2004, Insperger and Stépán developed the semi-discretisation method (SDM) (Insperger and Stépán 2002, 2004). Here, the delayed terms and the time periodic coefficients of the governing time periodic DDE are discretized, while the actual time domain terms and their derivatives are left in the original form. This approximation provides a series of ordinary differential equations that can be solved in each semi-discretization step. Solution of these equations with matching initial conditions results in a finite dimensional Floquet transition matrix. If the eigenvalues, the characteristic multipliers of this matrix, are in 
modulo less than 1 then the process is stable, otherwise, it is unstable and chatter arises. The predictive capabilities of SDM are satisfactory, but the computation is generally time-consuming since the number of discretization intervals required for good accuracy is high. The methods described above, perform the analysis on deterministic models which are usually simplified and inaccurate.

Another issue is that the stability lobe diagrams are based on the frequency response function of the system. An instrumented hammer is used to excite the system and the response is measured with the aid of an accelerometer. The measured response is then exported to additional software for mathematical computations to create stability diagrams. Model parameters uncertainties due to this modal testing procedure can also lead to discrepancies between the real system behavior and the theoretical model. Moreover, a stability lobe diagram is only accurate for one specific tool, holder, spindle, machine, and workpiece combination, so one has to make the hammer test for each combination.

Because of these reasons, there is still a need for in-process detection methods to predict and avoid chatter frequencies during milling processes. There are several setup types for measuring the vibrations on rotating machines. Since accelerometers cannot be applied on the spindle shaft itself, the first option is to measure the vibration transmitted from the spindle into a nonrotating part. Noncontact position transducers, such as capacitive and inductive displacement sensors are possible measurement methods but these kinds of sensors are limited to be very close to the rotating part. Other noncontact measurement devices such as laser distance triangulation sensors (LDS), TV-holography, laser doppler vibrometers (LDV), or laser interferometer (LI) may be used. The most sensitive, accurate, and precise optical device for measuring distance or displacement is the laser interferometer. This apparatus uses interference to make measurements of distances. These vibrometers are expensive devices. Errors occur when the contrast of the interferometer signal is not perfect and when the phase-shifted signal is not shifted by exactly $90^{\circ}$. Besides, the price of these devices is far higher than the price of the device shown here. An inexpensive method would be to detect chatter with the help of a microphone (Schmitz 2003). During chatter, besides the tooth path frequencies other frequencies arise. Although these would be detectable with a microphone, in a laboratory or a machine shop a microphone could collect a large amount of noise; one would have to filter these out permanently, and this would make the measurements more difficult, and less reliable.

The objective of this project is to build a low cost optical measurement apparatus that is able to record the displacement of a rotating mill bit during a cutting process, and to determine at what spindle speed and cutting depth the process becomes unstable. During a stable milling process, the data should show only the frequencies associated with the teeth passing through the workpiece. During an unstable cut, the characteristics of the graphs should show a noticeable change, where additional vibrational frequencies associated with chatter will appear. This is discussed in section 5 .

\section{MEASUREMENT SETUP}

Figure 1 shows the optical setup mounted on the lathe. The basic parts of the setup are labeled in the figure. It consist of several mounting brackets, laser source, 


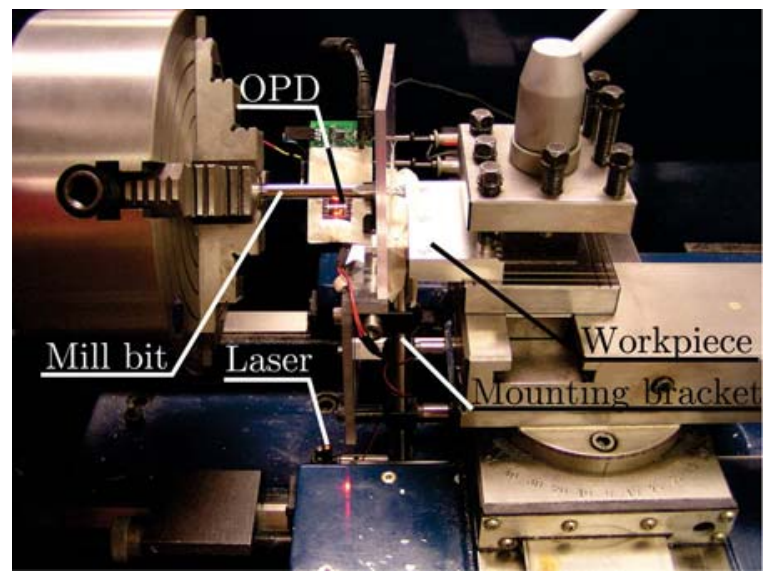

Figure 1. Optical measurement setup mounted on the lathe.

an optical position detector, and signal processing circuit. In Figure 2, one can see a schematic from the hall setup and the relative position of the laser and the optical position detector.

\subsection{Laser}

A micro LDM laser diode from Edmund optics (part no.: NT57-101) was used as the illumination source. It included automatic power control circuits, focusable optics, and had a visible red light output at $653 \mathrm{~nm}$. Its focus range was $50 \mathrm{~mm}$ to $\infty$ with a beam size at the focus of less than $50 \mu \mathrm{m}$.

\subsection{Optical Position Detector}

The laser beam was directed onto the mill bit. The reflected beam was projected onto an optical position detector (OPD) (see Figures 2a and 2b). The OPD was from Hamamatsu (part no.: S5991-01). The active area of the OPD was $9 \times 9 \mathrm{~mm}$. The theoretical position resolution of the OPD was $\Delta R_{t}=1.5 \mu \mathrm{m}$, but during the measurement it turned out that the real position sensitivity was only $\Delta R_{r}=70 \mu \mathrm{m}$. The reason for that was probably that the reflected beam couldn't focus as a small dot on the OPD.

\subsection{Milling Bit Selection}

For the measurements, two mill bits were used. These were long single end mills from Micro 100 Tool Corporation (part no.: GLR-312-2), and from T \& M Grinding with a diameter of $D=5 / 16$ in, two helical flutes, and an overall length of $L_{f}=4 \mathrm{in}$. The material properties of the bits are shown in Table 1. Because of the flutes of the bits, the beam could not be reflected from their tip because this would have changed the angle of the reflection constantly, and would have caused dificulties during the measurements. Because of the curvature of the bits, 


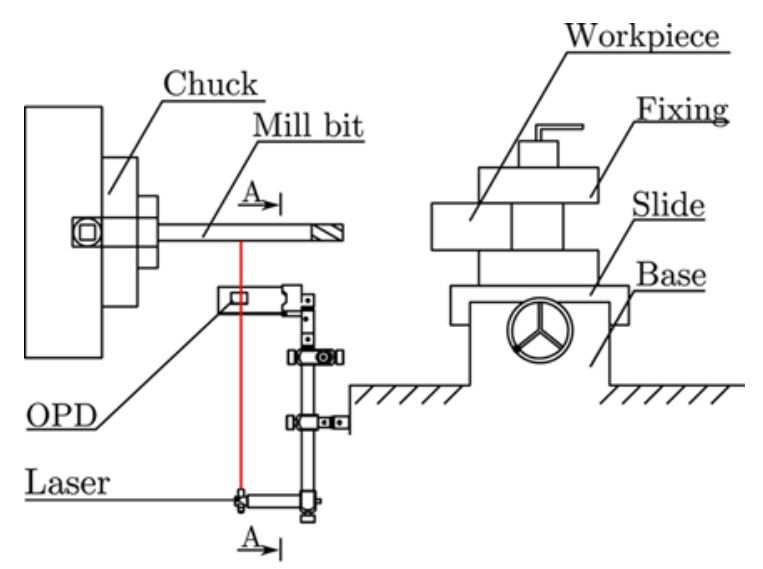

(a)

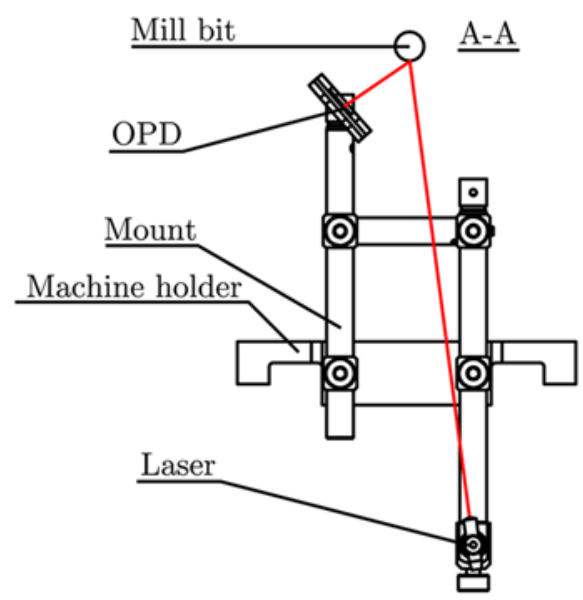

(b)

Figure 2. Optical measurement setup. (a) Schematic of the optical measurement setup (front view) and (b) Schematic of the optical measurement setup (side view).

the cross-section of the treflected laser beam was bigger than the area of the OPD, so the neck parts of the bits had to be polished to lower the cross-section size of the laser beam to get specular reflection conditions and reduce the specke noise. The eccentricity of a turning bit was under $e x=25 \mu \mathrm{m}$. Due to laboratory

Table 1. Material properties of the used mill bits

\begin{tabular}{lcc}
\hline & Micro 100 & T \& M grinding \\
\hline$E$ (Young's Modulus) Gpa & 139.3 & 580 \\
$\rho$ (Density) kg/m $\mathrm{m}^{3}$ & 14,554 & 14,450 \\
$\nu$ (Poisson's R.) & 0.3 & Unknown \\
\hline
\end{tabular}


limitation, a lathe (Enco Type: \# 308-0233) was used to emulate a milling machine by interchanging the positions of the workpiece and the working tool. The mill bit was fixed into the spindle of the lathe and the probe, and an aluminum brick was placed on the place of the cutting knife. Measurements were made with spindle speeds of $\Omega=385,510,585,770,900,1,170$, and, $1,800 \mathrm{rpm}$, with axial cutting depths of $a_{p}=1-6 \mathrm{~mm}$ with a constant increase of $0.5 \mathrm{~mm}$, and with a constant from the lathe-controled feed rate of $f_{z}=0.078=\mathrm{mm} /$ tooth. Due to the limitations of the lathe, no high speed cutting processes were investigated.

A digital oscilloscope (Link Instruments Type: DSO - 8500) was connected to a laptop (Dell Latitude D610). The OPD was connected to the oscilloscope. The frequency bandwidth of the optical system was equal with the bandwidth of the oscilloscope, and this was $b w=100 \mathrm{MHz}$. A manufacturer-suggested current sensing circuit was used to measure the position of the reflected beam (see Figures $2 a$ and $2 b$ ). The position of the beam was determined according to the following formulas.

$$
\begin{aligned}
& \frac{\left(I_{2}+I_{3}\right)-\left(I_{1}+I_{4}\right)}{I_{1}+I_{2}+I_{3}+I_{4}}=\frac{2 x}{L} \\
& \frac{\left(I_{2}+I_{4}\right)-\left(I_{1}+I_{3}\right)}{I_{1}+I_{2}+I_{3}+I_{4}}=\frac{2 y}{L},
\end{aligned}
$$

where $I_{i}$ values are the currents on the four subareas of the OPD, $x$ and $y$ are the perpendicular deflections on the OPD, and $L$ is $L=10 \mathrm{~mm}$ for S5991-01. These formulas were provided from the manufacturer and can be dound on the data sheet of the OPD.

Two output signals were collected on the two channels of the oscilloscope connected to the laptop through a usb data port, and processed with a Matlab program. The laser and the OPD were attached to mounting tools from Thorlabs; these posts and clamps are specifically designed for optical measurements. To avoid constructing a separate stand for the laser and the OPD, they were attached through mounting posts and clamps from Thorlabs directly to the main slide of the lathe. The posts and clamps are designed for optical measurements. Because of this mounting method, it had to be checked to see if the vibrations of the lathe itself would influence the measurement. Tests with rotating spindle but without cutting showed that the vibrations of the machine were negligible compared to the vibrations of the mill bit during cutting. Figure $2 b$ shows the relative position of the laser and the OPD to the mill bit.

\section{MEASUREMENT PRINCIPLES}

Using simple ray tracing, one can show that

$$
\Delta x=r \beta
$$

where $r$ is the radius of the beam. Figure 3 shows a schematic of how the angle of the reflected beam changes if the bit moves. The position of the laser doesn't change during the movement of the bit, so the angle of incident after the movement will be 


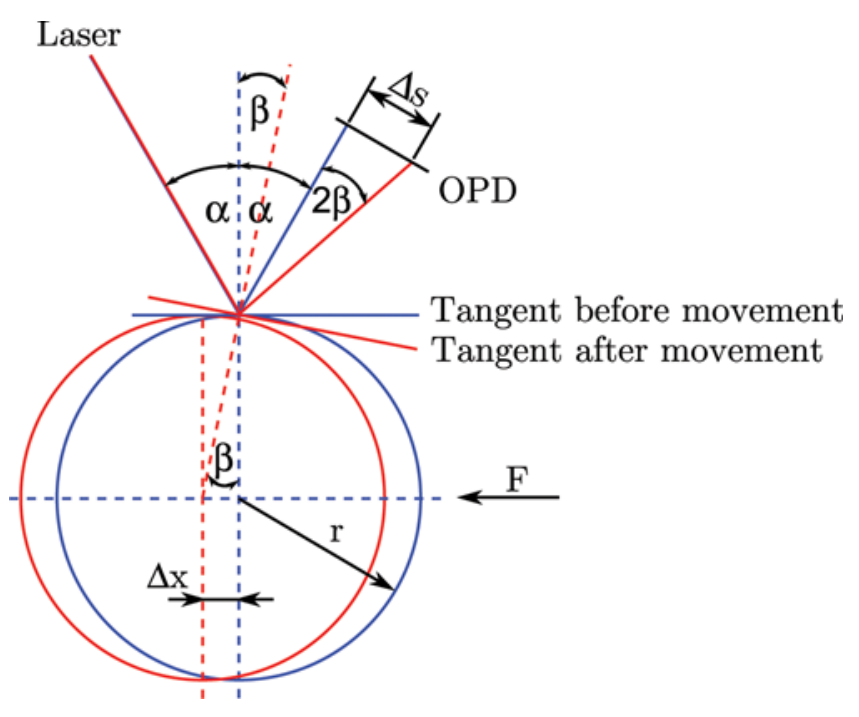

Figure 3. Laser beam reflection diagram.

$\alpha+\beta$. It is easy to see that the angle between the reflected rays before and after the movement will be $2 \beta$. For small deflections $\Delta s$ will be

$$
\Delta s=2 t \beta
$$

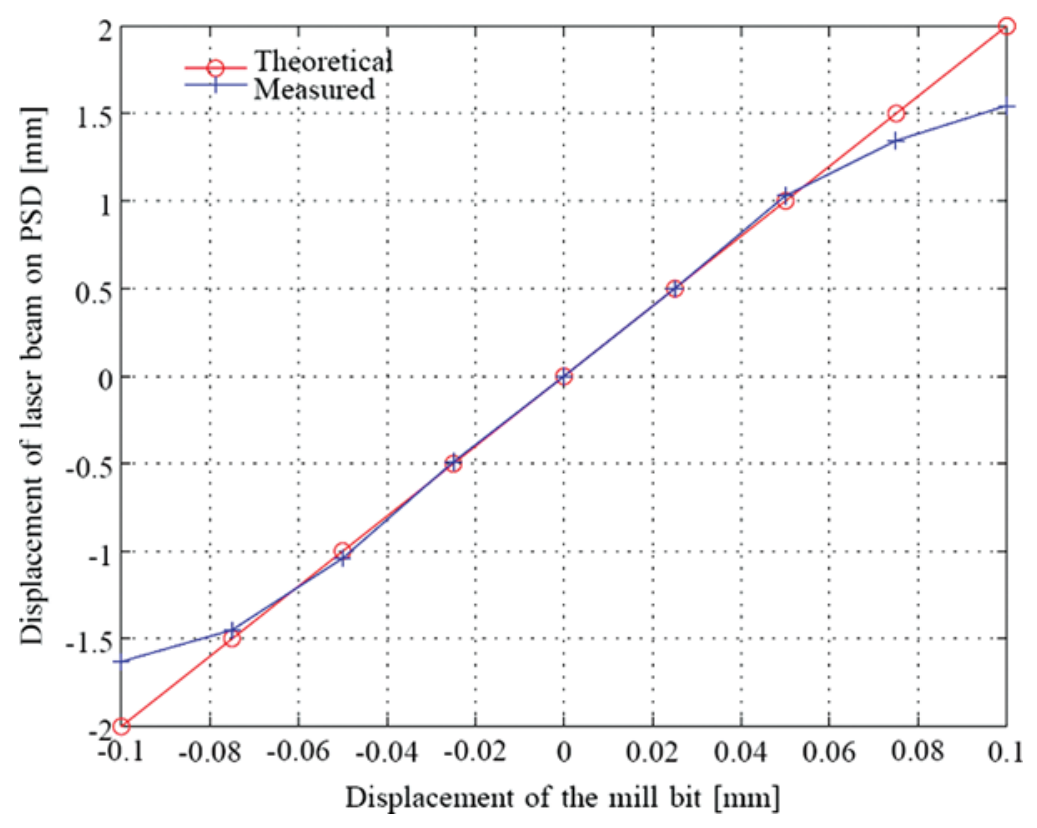

Figure 4. Connection between $\Delta x$ and $\Delta s$. 
Therefore, the connection between the deflection of the bit and the reflected light spot on the OPD will be

$$
\Delta s=\frac{2 t}{r} \Delta x
$$

The bit size was chosen according to its known material properties, such that for an estimated reaction force of $F=80 \mathrm{~N}$, (Mann et al. 2002), the maximal deflection on the OPD (Type: 5990-01) is $\Delta s_{\max }=4 \mathrm{~mm}$. Validation of Eq. (5) was performed using a micro-positioner moving the bit with prescribed displacement. The calibration showed that the definition in Eq. (5) of the connection between the bit movement and the reflected beam movement on the OPD is precise enough. Moreover, it also shown that the measured displacement on the OPD differs from the theoretical (Figure 4), this had to be considered during the calculations and programming of the Matlab code. The diameter of the chosen bit was $D=5 / 16$ in and the distance between the bit and the OPD was $t=40 \mathrm{~mm}$. This means an amplification of the bit displacement to the displacement of the reflected beam on the OPD is by 10 . With these parameters, the difference between the displacement on the OPD due to the calibration (in the measured point $x=0.025 \mathrm{~mm}, y=$ $0.49 \mathrm{~mm}$ ) and the counted displacement was about $3 \%$.

\section{MEASUREMENTS}

First, the natural frequency of the bit (from T \& M Grinding) was measured. An estimation was made according to the Rayleigh method. The first mode can be estimated with the following expression.

$$
\alpha_{1}^{2}=\frac{I E \int_{0}^{l} Y^{\prime \prime^{2}}(x) d x}{\rho S \int_{0}^{l} Y^{2}(x) d x}\left[\frac{1}{s^{2}}\right]
$$

where $I$ is the inertia of moment of the bit, $E$ is the Young's modulus, $\rho$ is the density, $S$ is the area of the cross-section, and $Y$ is an estimation function. This is a simple polynomial function $Y=x^{3}+a_{2} x^{2}+a_{1} x+a_{0}$. The constants $a_{1}, a_{2}$, and $a_{3}$ were calculated due to the edge conditions $Y(0)=0, Y^{\prime}(0)=0$, and $Y^{\prime \prime}(l)=0$, where $l$ is the total length of the beam. According to the calculations, the first mode of the bit was $f_{1}=\alpha_{1} / 2 \pi=1,109.9 \mathrm{~Hz}$. The calculation was also checked with a simple FEM model created in Ansys. The calculated first mode was here $f_{1}=1,125.7 \mathrm{~Hz}$. The sampling frequency with the oscilloscope was $f_{s}=1 \mathrm{MHz}$. The measured self-vibration was $f_{1}=1,162 \mathrm{~Hz}$, which compares well with the estimated values. The measurements were repeated during cutting. During the processes, no lubricants were used. The laser and the OPD was covered with a plexi plate, so the chips produced during cutting could not damage them and could not affect the measurements.

So-called full immersion cutting or slotting was done. Here, the immersion ratio was $a / D=1$, where $a$ is the radial immersion and $D$ is the diameter of the bit. The experiments did not show chatter on the frequency spectrum. A second set of measurements was made with an immersion ratio of $a / D=0.25$ and $a / D=0.5$. All of these measurements were made as down milling cutting processes. The parameters 
of the measured points are described in section 2 and subsection 2.3, the only difference is that the maximal cut depth $a_{p}$ was reduced to $4 \mathrm{~mm}$. Besides collecting the data, photographs were made of the work pieces and the cuts. Stability maps were made according to the stored data and according to the photos, and these were compared afterwards. The stored data was processed with a Matlab program calculating the Fourier (FFT) and power spectrum density (PSD) graphs. The PSD graphs showed characteristic changes when a cut was unstable. The interpretation of these results requires an in-depth analysis of the chatter phenomenon.

\section{ANALYSIS OF CHATTER}

\subsection{Mechanical Model}

The mathematical model describing a milling process is a delay-differential equation (DDE) with infinite dimensional state space. For continuous cutting operations, like turning, the governing equation is autonomous and the stability conditions can be given in closed form. The study of nonlinear phenomena in the cutting process showed that the chatter frequencies are related to unstable periodic motions about the stable stationary cutting, i.e., a so-called subcritical Hopf bifurcation occurs, as proven experimentally by Shi and Tobias (1984), and later analytically by Stépán and Kalmár-Nagy (1997).

The theoretical explanation in this section is based on Insperger et al. (2006). The standard 2-DOF (degree of freedom) mechanical model of end milling is shown in Figure 5. The tool is assumed to be flexible relative to the rigid workpiece. The

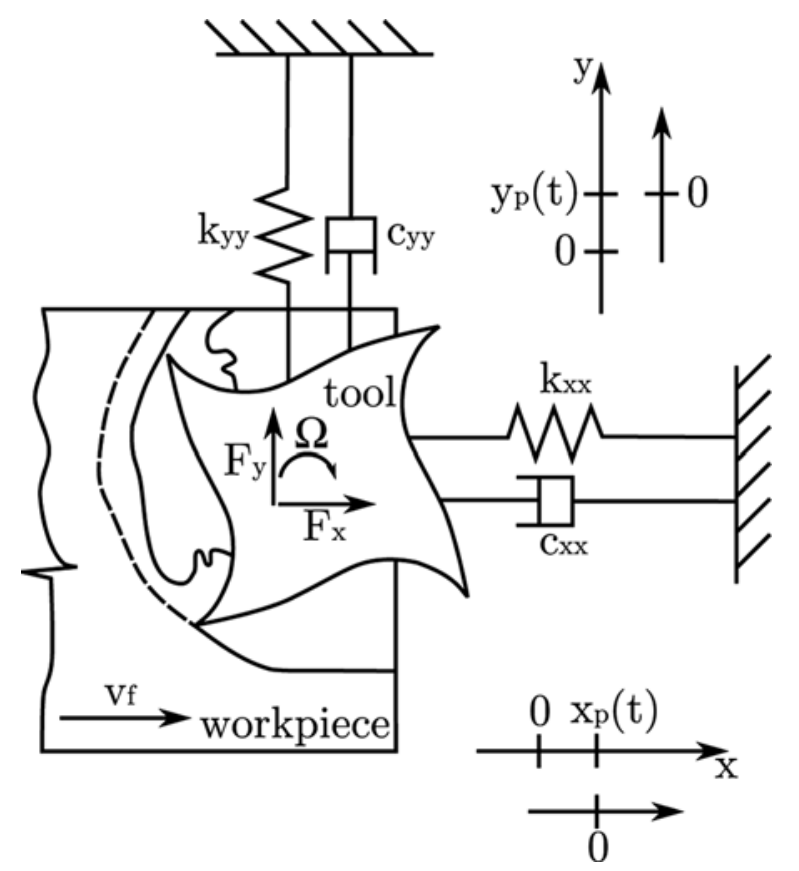

Figure 5. Schematic of 2-DOF milling model. 
2-DOF oscillator is excited by the cutting force $\mathbf{F}(t)$. The governing equation has the following form.

$$
\mathbf{M} \ddot{\mathbf{x}}(t)+\mathbf{C} \dot{\mathbf{x}}(t)+\mathbf{K} \mathbf{x}(t)=\mathbf{F}(t)
$$

where

$$
\mathbf{x}(t)=\left[\begin{array}{l}
x(t) \\
y(t)
\end{array}\right], \quad \mathbf{F}(t)=\left[\begin{array}{l}
F_{x}(t) \\
F_{y}(t)
\end{array}\right]
$$

The $x$ and $y$ components of the cutting force are given as

$$
\begin{aligned}
& F_{j x}(t)=F_{j t}(t) \cos \phi_{j}(t)+F_{j n}(t) \sin \phi_{j}(t) \\
& F_{j y}(t)=-F_{j t}(t) \sin \phi_{j}(t)+F_{j n}(t) \cos \phi_{j}(t) .
\end{aligned}
$$

where $F_{j t}(t)$ and $F_{j n}(t)$ are the normal and tangential forces acting on $j$ th tooth, respectively, and $\phi_{j}(t)$ is the angular position of the $j$ th cutting edge. Let the feed per tooth be denoted by $f_{z}=v_{f} \tau$ where $v_{f}$ is the feed speed and $\tau=60 /(N \Omega)$ is the tooth passing period. If $f_{z} \ll R$, where $R$ is the radius of the tool and $A$ and $B$ are the horizontal and vertical distances of the real previous and real current tooth passes, then the instantaneous chip thickness can be expressed according to Figure 6 as

$$
\begin{aligned}
h(t) & \approx A \sin \phi_{j}(t)+B \cos \phi_{j}(t) \\
& =\left[f_{z}+x(t-\tau)-x(t)\right] \sin \phi_{j}(t)+[y(t-\tau)-y(t)] \cos \phi_{j}(t)
\end{aligned}
$$

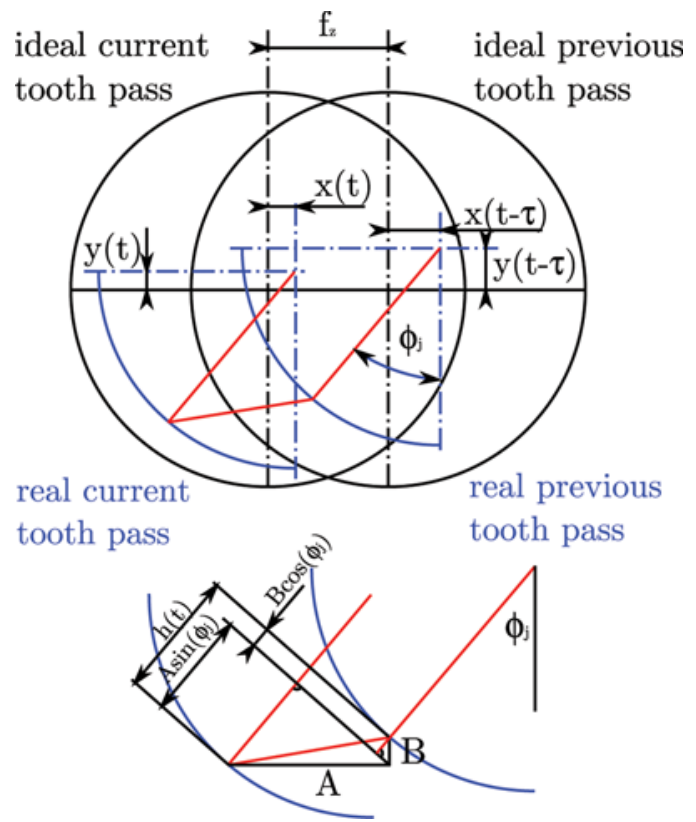

Figure 6. Chip thickness. 
The resultant cutting forces are the sum of the forces acting on the teeth.

$$
\begin{aligned}
F_{x}(t) & =\sum_{j=1}^{N} F_{j x}(t) \\
& =\sum_{j=1}^{N} g\left[\phi_{j}(t)\right]\left[K_{t} \cos \phi_{j}(t)+K_{n} \sin \phi_{j}(t)\right] a_{p} h(t) \\
F_{y}(t) & =\sum_{j=1}^{N} F_{j y}(t) \\
& =\sum_{j=1}^{N} g\left[\phi_{j}(t)\right]\left[-K_{t} \sin \phi_{j}(t)+K_{n} \cos \phi_{j}(t)\right] a_{p} h(t)
\end{aligned}
$$

where $g\left[\phi_{j}(t)\right]$ is a screen function, which is 1 when the $j$ th tooth is cutting and 0 when not. $K_{t}$ and $K_{n}$ are the linear tangential and linear normal cutting coefficients. So, the equation of motion can be written in the following form.

$$
\mathbf{M} \ddot{\mathbf{x}}(t)+\mathbf{C} \dot{\mathbf{x}}(t)+\mathbf{K} \mathbf{x}(t)=a_{p} \mathbf{H}(t)[\mathbf{x}(t-\tau)-\mathbf{x}(t)]+\mathbf{G}(t)
$$

where $\mathbf{H}(t)$ is the force variation matrix (Altintas and Budak 1995a). $\mathbf{G}(t)$ is the stationary cutting force vector

$$
\begin{aligned}
& G_{t}(x)=a_{p} f_{z} H_{x} x(t) \\
& G_{t}(y)=a_{p} f_{z} H_{y} x(t)
\end{aligned}
$$

\subsection{Forced and Self-Excited Motion of the Tool}

The motion of the workpiece is decomposed in the form:

$$
\mathbf{x}(t)=\mathbf{x}_{\mathbf{p}}(t)+\xi(t)=\left[\begin{array}{l}
x_{p}(t) \\
y_{p}(t)
\end{array}\right]+\left[\begin{array}{l}
\xi(t) \\
\eta(t)
\end{array}\right]
$$

where $\mathbf{x}_{\mathbf{p}}(t)=\mathbf{x}_{\mathbf{p}}(t-\tau)$ is the forced chatter free motion of the tool, and $\xi(t)$ is a perturbation corresponding to the self-excited vibrations of the tool. Substitution of Eq. (16) into Eq. (13) results in

$\mathbf{M} \ddot{\mathbf{x}}_{\mathbf{p}}(t)+\mathbf{C} \dot{\mathbf{x}}_{\mathbf{p}}(t)+\mathbf{K} \mathbf{x}_{\mathbf{p}}(t)+\mathbf{M} \ddot{\xi}(t)+\mathbf{C} \dot{\xi}(t)+\mathbf{K} \xi(t)=a_{p} \mathbf{H}(t)[\xi(t-\tau)-\xi(t)]+\mathbf{G}(t)$

For the ideal case, when no chatter arises, $\xi(t)=0$, and the motion is described by $\mathbf{x}(t)=\mathbf{x}_{\mathbf{p}}(t)$, the corresponding equation of motion is an ordinary differential equation.

$$
\mathbf{M} \ddot{\mathbf{x}}_{\mathbf{p}}(t)+\mathbf{C} \dot{\mathbf{x}}_{\mathbf{p}}(t)+\mathbf{K} \mathbf{x}_{\mathbf{p}}(t)=\mathbf{G}(t)
$$


For linear stability analysis the variational system of Eq. (13) is determined around the periodic chatter-free motion $\mathbf{x}_{\mathbf{p}}(t)$. Equations (17) and (18) imply the equation

$$
\mathbf{M} \ddot{\xi}(t)+\mathbf{C} \dot{\xi}(t)+\mathbf{K} \xi(t)=a_{p} \mathbf{H}(t)[\xi(t-\tau)-\xi(t)]
$$

If the cutting process is stable, the component $\xi(t)$ corresponds to the chatter signal decays, and the tool moves according to the periodic chatter-free motion described by $\mathbf{x}_{\mathbf{p}}(t)$. If the process is unstable, $\xi(t)$ increases exponentially, and the resultant motion $\mathbf{x}(t)=\mathbf{x}_{\mathbf{p}}(t)+\xi(t)$ will also obtain exponential growth.

For unstable machining, the nonlinear phenomena also plays an important role in the system's dynamics. Due to its large vibrations, the tool leaves the cut and the cutting force instantly drops to zero. This nonlinearity actually stops the exponential amplitude growth so that in practice, the tool vibrations during unstable machining still have a finite amplitude (Stépán et al. 2005).

The linear stability of the milling process is described by Eq. (19). The stability analysis of this time periodic DDE can be determined by the semidiscretization method (Insperger et al. 2003). The critical multipliers can be located three ways.

1) They are a complex pair located on the unit circle, $|\mu|=1$. This case is topologically equivalent to the Hopf bifurcation of autonomous systems.

2) $\mu=1$. The associated bifurcation is topologically equivalent to the saddle-node bifurcation of autonomous systems and is called a period one bifurcation.

3) $\mu=-1$. This case is called period doubling or flip bifurcation. There is no topologically equivalent type of bifurcation for autonomous systems.

According to Davies et al. (2002), in milling processes only cases 1) and 3) can be observed. First, when $\mu$ is a complex pair then characteristic frequencies arise in the vibration signal. These frequencies can be defined as

$$
f_{H}^{n}=\left\{ \pm \frac{\omega}{2 \pi}+n \frac{z \Omega}{60}\right\}[\mathrm{Hz}] \quad n=\ldots,-1,0,1, \ldots
$$

and refer to the secondary Hopf bifurcation. Second, when $\mu<-1 ; \mu \in \mathbf{R}$, then another type of characteristic frequencies arise in the measured signal. These frequencies can be defined as

$$
f_{P D}^{n}=\frac{z \Omega}{120}+n \frac{z \Omega}{60}[\mathrm{~Hz}] \quad n=\ldots,-1,0,1, \ldots
$$

$\omega=\operatorname{Im}(\ln \mu) / \tau$ where $\mu$ is the characteristic multipliers, $z$ is the number of teeth, and $\Omega$ is the spindle speed of the machine.

During a stable milling operation, the excited frequencies are related to all components of $\mathbf{x}(t)$ defined by Eq. (16). The periodic motion $\mathbf{x}_{p}(t)$ contains the characteristic frequencies defined as

$$
f_{T P E}^{n}=\frac{n z \Omega}{60}[\mathrm{~Hz}] \quad n=1,2,3, \ldots
$$

Because of the runout of the bit the period, doubling frequencies $\left(f_{P D}^{n}\right)$ turn into tooth path frequencies $\left(f_{T P E}^{n}\right)$ because the principal period changes from $\tau=z(\Omega / 60)$ 
to $T=z \tau$ (Insperger et al. 2008). Runout is a phenomenon where the loads on the cutting teeth of the mill bit are not equal. This comes out of the asymmetry of the multiflute bits.

Run out typically arises in machining with bits that have more than one flutes because the tool is never ideally symmetric. During a stable process, peaks should be visible only at the $f_{T P E}^{n}$ and $f_{P D}^{n}$ frequencies. The expected changes on the graphs during an unstable cut were that peaks arise not only at these frequencies but
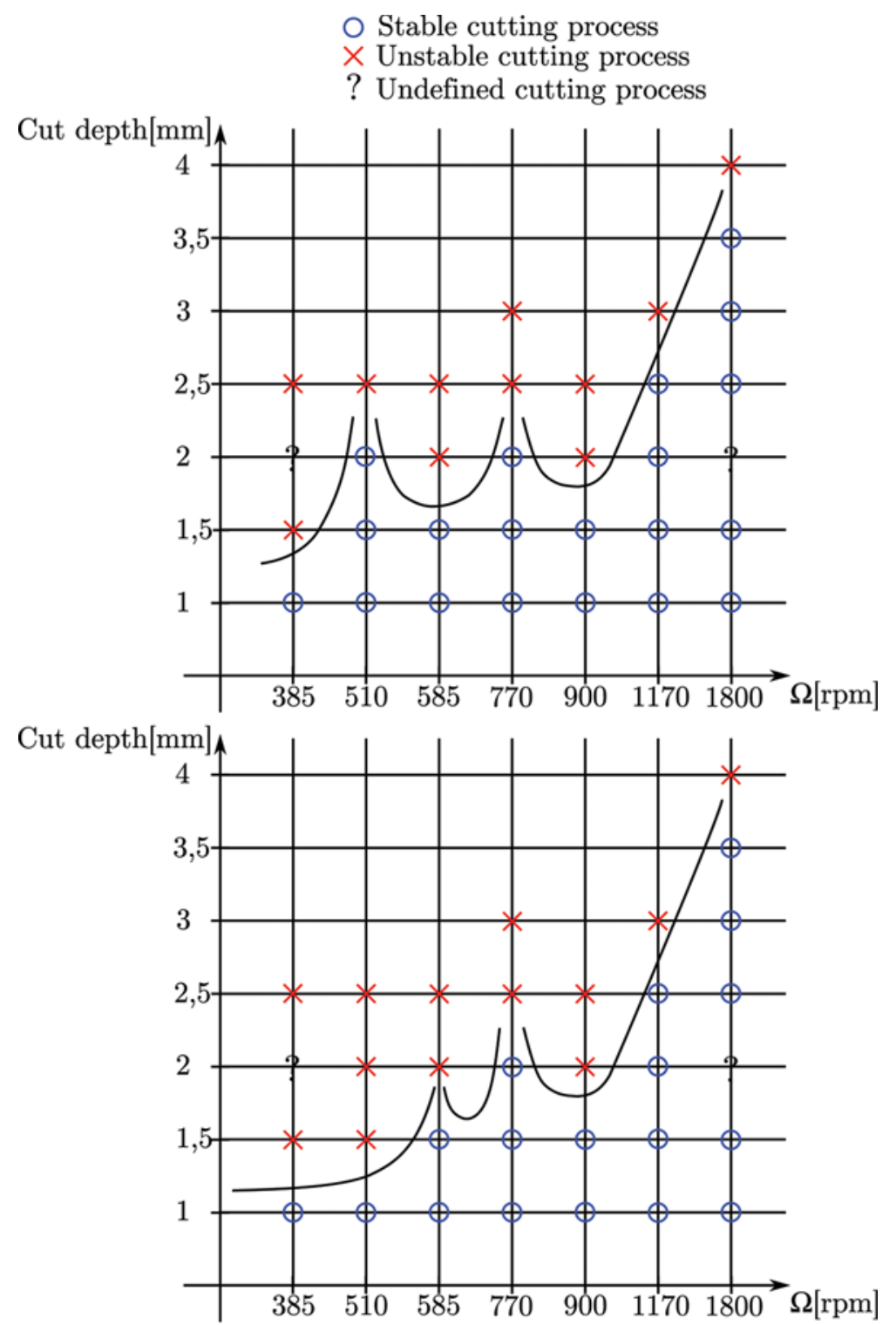

Figure 7. Stability maps according to data (top) and according to photos (bottom). 
Table 2. Characteristic frequencies

\begin{tabular}{lccc}
\hline$\Omega(\mathrm{rpm})$ & \multicolumn{3}{c}{$f_{T P E}^{n}(\mathrm{~Hz})$} \\
\cline { 2 - 4 }$n$ & 1 & 2 & 3 \\
\hline 385 & 13.7 & 27.4 & 41.1 \\
510 & 18.3 & 36.6 & 54.9 \\
585 & 20.9 & 41.7 & 62.6 \\
770 & 27.4 & 54.7 & 82.1 \\
900 & 31.7 & 63.3 & 95 \\
1,170 & 41.6 & 83.3 & 124.9 \\
1,800 & 63.1 & 126.1 & 189.2 \\
& & $f_{P D}^{n}(\mathrm{~Hz})$ & \\
385 & 20.5 & 34.3 & 48 \\
510 & 27.5 & 45.7 & 64 \\
585 & 31.3 & 52.2 & 73 \\
770 & 41 & 68.4 & 95.8 \\
900 & 47.5 & 79.2 & 110.8 \\
1,170 & 62.5 & 104.1 & 145.7 \\
1,800 & 94.6 & 157.7 & 220.7 \\
\hline
\end{tabular}

also in between those. Contrary to this, we observed that during an unstable cut the $f_{P D}^{n}$ frequencies disappeared. Although the changes on the PSD graph of an unstable cut related to a stable cut were not expected ones, the dissapearance of the $f_{P D}^{n}$ frequencies is ovious, so the observations of the PSD graphs still give reliable information about the stability of a cutting process.

The first map was made according to $a / D=0.5$. For these, the comparison between photographs and vibration frequency peaks showed some differences.

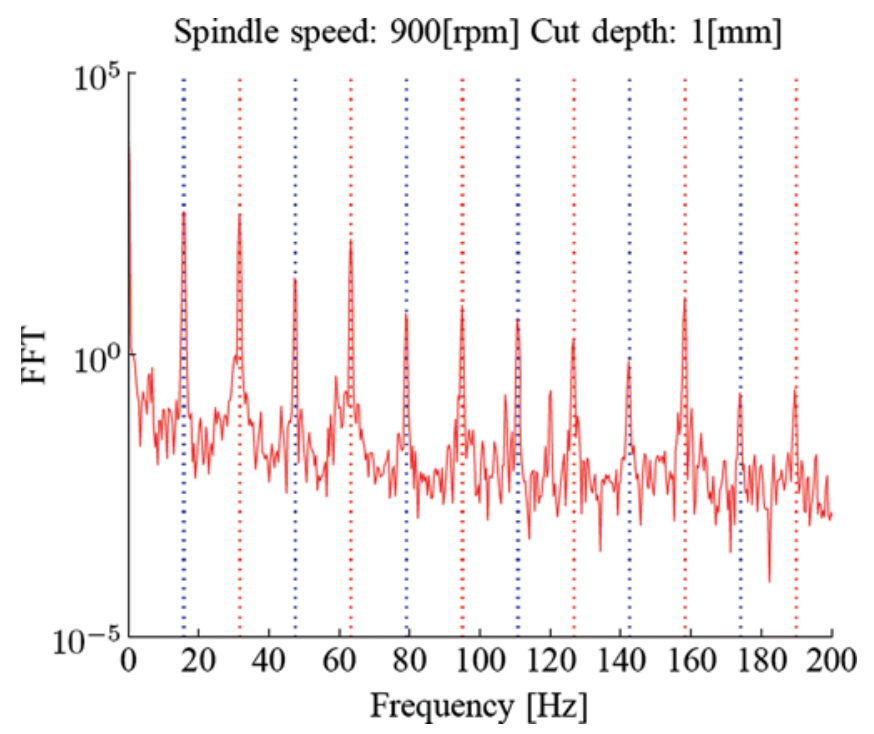

Figure 8. Power spectrum graph of a stable cut. 


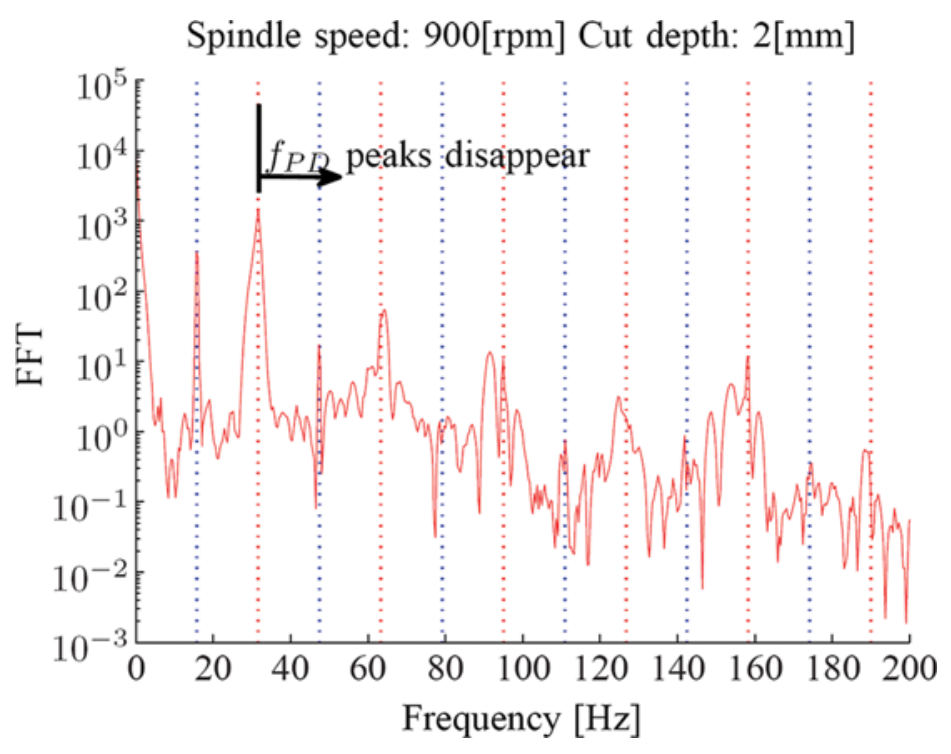

Figure 9. Power spectrum graph of an unstable cut.

The graphs didn't match each other completely. This was because the chosen sampling frequency was wrong. The oscilloscope used is able to store 524,200 points. The chosen sampling frequency was $f_{s}=50 \mathrm{KHz}$ and $f_{s}=100 \mathrm{KHz}$. At lower spindle speeds, this caused a too short measuring window and the unstable vibrations weren't recorded. Figure 7 shows the maps made according to the measurements and the photos. The difference is at $\Omega=510 \mathrm{rpm}, a_{p}=1.5 \mathrm{~mm}$, $\Omega=510 \mathrm{rpm}$, and $a_{p}=2 \mathrm{~mm}$ on the two graphs. The boundary lines for stable and unstable processes are estimations and sketches, no calculations were done to determine the stability lobes. Figures 8 and 9 show the PSD graphs of the power spectrum of the measured displacement. The red and blue dotted vertical lines show

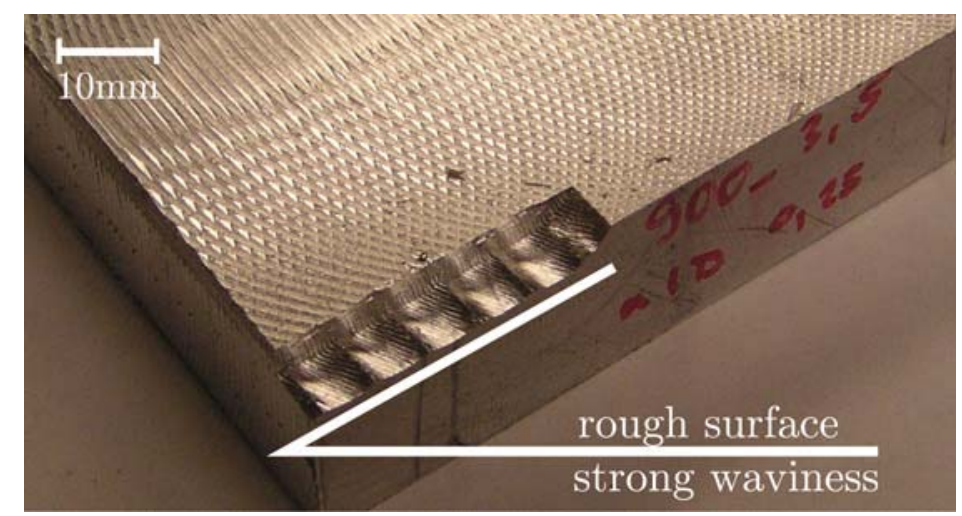

Figure 10. Cut surfaces of an unstable cut. 
the values for the $f_{T P E}^{n}$ and $f_{P D}^{n}$. A few values are listed in Table 2. The basic difference between Figures 8 and 9 is the arising peaks at the $f_{P D}^{n}$ frequencies. During an unstable cut, these peaks disappear on the graphs. In Figure 11 one can see the cuts on the work pieces. The parameters are the same as for the PSD graphs in Figures 8 and 9. One can see that with a cut depth of $1 \mathrm{~mm}$ the cut is stable and straight, but with a cut depth of $2 \mathrm{~mm}$ the cut is unstable, i.e., it is wavy. That is because of the self-excited vibrations.

Going deeper into the unstable region on the stability map, the frequency of leaving the cut increases. One can see in Figure 10 that the waves in the cut are shorter than in Figure 11. The best results were obtained for $\Omega=385,510,585 \mathrm{rpm}$ a sampling frequency of $f_{s}=20 \mathrm{KHz}$, for $\Omega=770,900 \mathrm{rpm} f_{s}=50 \mathrm{KHz}$, and for $\Omega=1,170,1,800 \mathrm{rpm} f_{s}=100 \mathrm{KHz}$. Measurements were made with this frequencies at an immersion ratio of $a / D=0.25$.

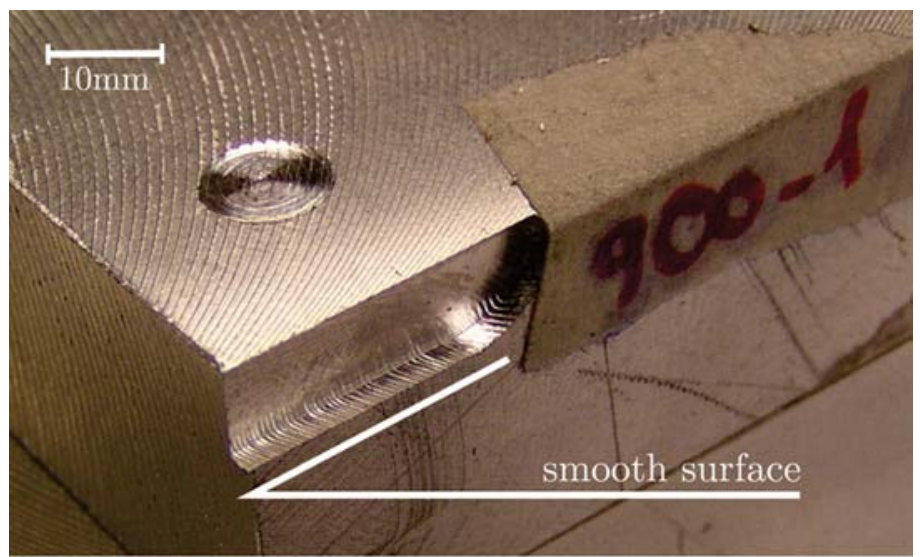

(a)

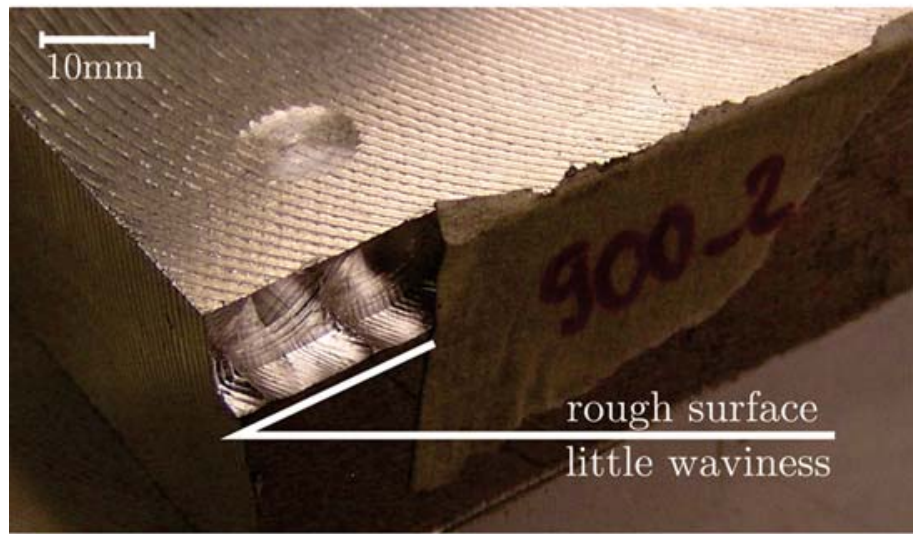

(b)

Figure 11. Cut surfaces of a stable and an unstable cut. (a) $900 \mathrm{rpm}, 1 \mathrm{~mm}$ cut depth and (b) $900 \mathrm{rpm}$, $2 \mathrm{~mm}$ cut depth. 


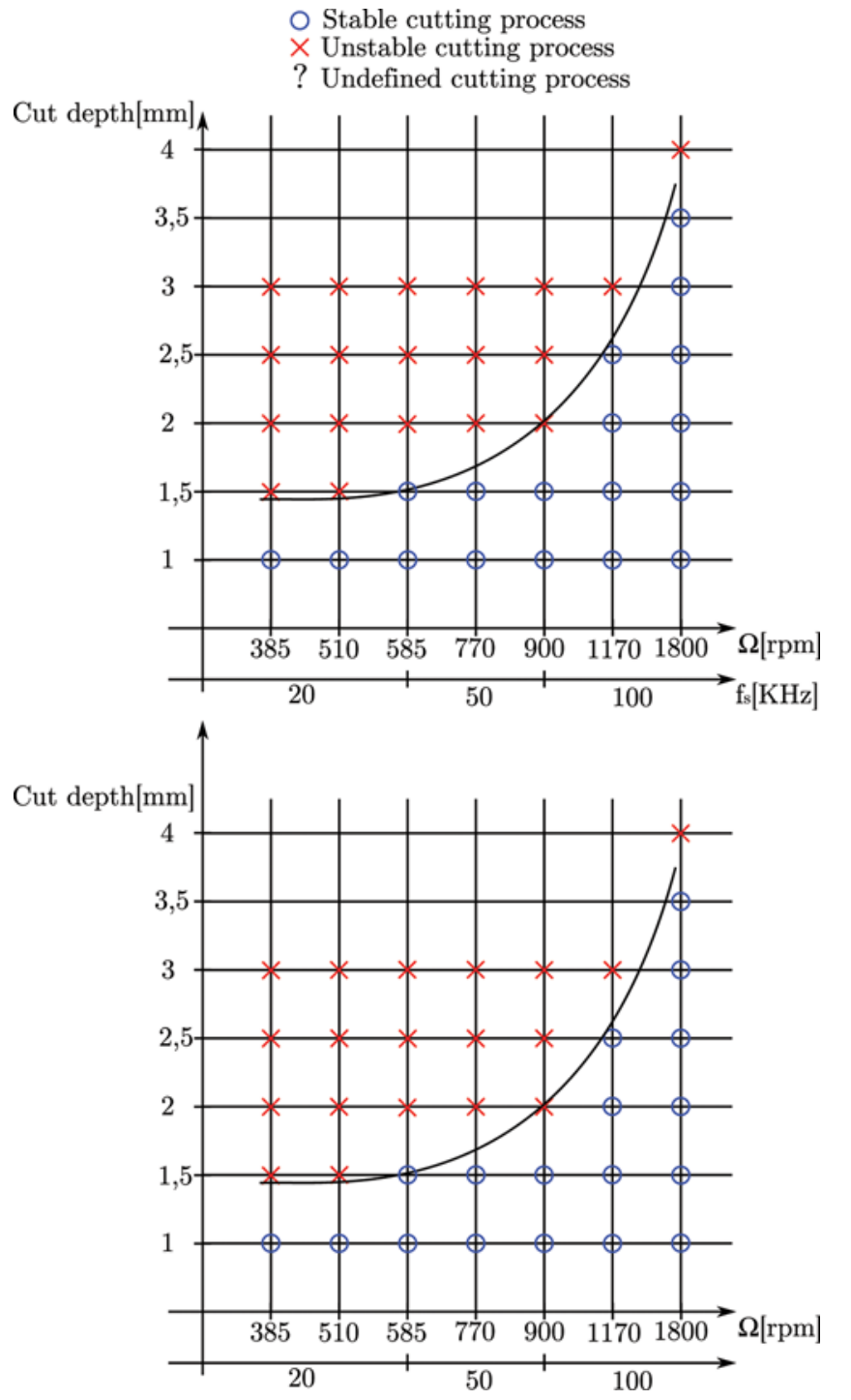

Figure 12. Stability maps according to data (top) and according to photos (bottom).

The comparison of the stability maps based on the recorded data and the photos were satisfactory. The graphs matched each other at all points. Figure 12 shows the two maps.

\section{CONCLUSION}

The real time measurements and the comparison of the cut surfaces and the recorded data show that the setup is able to detect chatter vibrations based on PSD analysis. For spindle speeds of $\Omega=385,510,585 \mathrm{rpm}$, the best sampling frequency 
was $f_{s}=20 \mathrm{KHz}$. The oscilloscope was able to store a maximum number of 524,200 points. At a high sampling rate at these spindle speeds, the resulting measuring window was too short to capture the instability developement. For spindle speeds of $\Omega=770,900 \mathrm{rpm}$ the best sampling frequency was $f_{s}=50 \mathrm{KHz}$. For spindle speeds of $\Omega=1,170,1,800 \mathrm{rpm}$, the best sampling frequency was $f_{s}=100 \mathrm{KHz}$. A lower frequency caused too long of a measurement. After about $10 \mathrm{~s}$, the slide of the machine reached its limits, and the length of the measurement with $f_{s}=$ $50 \mathrm{KHz}$ was about $16 \mathrm{~s}$. Because of the two flutes on the bit and the runout during a stable cutting process, the $f_{T P E}^{n}$ peaks turned into $f_{P D}^{n}$ peaks like those in Figure 8. The measuring method can be adapted on a vertical CNC machine. Connecting a second laser, the horizontal deflections could also be detected and a two-dimensional deflection analysis can be done.

\subsection{Advantages of the System}

- The setup is quite flexible. The parts are not limited to be very close to the rotating bit, as in the case of the capacitive and inductive displacement sensors. One can mount the laser and the OPD onto the machine itself if the housing is stiff enough, or build a separate stand for them.

- It is not as sensible as the laser interferometer because its work principles are not based on the phase shift of the laser light.

- The setup itself is really simple; it is built only out of a few parts. The main elements are the laser and the OPD.

- Compared to most of the other devices, this setup is quite inexpensive.

\subsection{Disadvantages of the System}

- The surface where one wants to reflect the laser beam has to be polished, and in the case of a rotating mill, the roundness of the bit has to be ensured to avoid speckle noise.

- The OPD is sensible against all kinds of dirt and impurities. If the setup is built close to the bit, one has to cover it so that no chips of the workpiece fall onto it because this would damage the OPD.

- The device is not measuring the vibrations on the tool tip but at the neck part of a bit, so the results don't show the vibrations arising at the cutting point.

\section{ACKNOWLEDGMENTS}

This article is based on a thesis, written by Andras Eppel at the University of Arizona, Department of Aerospace and Mechanical Engineering. The project was supported by a bilateral grant from the U.S. Dept. of Education (\#P116J080016), the Atlantis program of the European Commission (2008-1767/001-001 CPT USMOBI), and by a grant from NSF (DUE\# 0633312).

\section{REFERENCES}

Altintas, Y. and E. Budak. 1995a. Chatter instability in milling systems with flexible rotating spindles - A new theoretical approach. ASME J. Manuf. Sci. Eng. 123(1):1-9. 
Altintas, Y. and E. Budak. 1995b. Analytical prediction of stability lobes in milling. Annals of the CIRP 44(1):357-362.

Campomanes, M. L. and Y. Altintas. 2003. An improved time domain simulation for dynamic milling at small radial immersions. Manufacturing and Engineering and Science 125(3):29-38.

Davies, M. A., J. R. Pratt, B. Dutterer, and J. Burns. 2002. Stability prediction for low radial immersion milling. Journal of Manufacturing Science and Engineering 124(2):217-225.

Insperger, T. and G. Stépán. 2002. Semi-discretization method for delayed systems. Int. J. Numer. Methods Eng. 55(5):503-518.

Insperger, T. and G. Stépán. 2004. Updated semi-discretization method for delayed systems. Int. J. Numer. Methods Eng. 61(1):117-141.

Insperger, T., J. Gradisěk, M. Kalveram, G. Stépán, K. Winert, and E. Govekar. 2006. Machine tool chatter and surface location error in milling. Journal of Manufacturing Science and Engineering 128(4):913-920.

Insperger, T., P. V. Bayly, G. Stépán, and B. P. Mann. 2003. Multiple chatter frequencies in milling processes. Journal of Sound and Vibration 262(2):333-345.

Insperger, T., B. P. Mann, T. Surmann, and G. Stépán. 2008. On the chatter frequencies of milling processes with runout. International Journal of Machine Tools \& Manufacture 48(10):1081-1089.

Mann, B. P., T. Insperger, P. V. Bayly, and G. Stépán. 2002. Stability of up-milling, and down-milling, part 2: experimental verification. International Journal of Machine Tools \& Manufacture 43(1):35-40.

Schmitz, T. L. (2003). Chatter recognition by a statistical evaluation of the synchronously sampled audio signal. Journal of Sound and Vibration 262(3):721-730.

Shi, H. M. and S. A. Tobias. 1984. Theory of finite amplitude machine tool instability. International Journal of Machine Tool Design and Research 24(1):45-69.

Stépán, G. and T. Kalmár-Nagy. 1997. Nonlinear regenerative machine tool vibration. Proceedings of the 1997 ASME Design Engineering Technical Conferences, Sacramento, California, pp. DETC97/VIB-4021 (CD-ROM).

Stépán, G., R. Szalai, B. P. Mann, P. V. Baily, T. Insperger, J. Gradisěk, and E. Govekar. 2005. Nonlinear dynamics of high-speed milling-analyses, numerics and experiments. ASME J. Vibr. Acoust. 127(2):197-203.

Tlustý, J., A. Polacek, C. Danek, and J. Spacek. 1962. Selbsterregte Schwingungen an Werkzeugmaschinen. Berlin: VEB Verlag Technik.

Tobias, S. A. 1965. Machine Tool Vibration. London: Blackie. 